\title{
Fuzzy Reliability Theory Based on Membership Function
}

\author{
Shshank Chaube ${ }^{*}$, S. B. Singh ${ }^{2}$ \\ ${ }^{1}$ Department of Engineering Mathematics \\ Graphic Era Hill University, Bhimtal Campus, Bhawali, Nainital, Uttarakhand-263136, India \\ ${ }^{2}$ Department of Mathematics, Statistics, and Computer Science \\ G. B. Pant University of Agriculture and Technology, Pantnagar, U. S. Nagar, Uttarakhand-263145, India \\ 1*chaube.shshank@gmail.com, 201rsurajbsingh@yahoo.com \\ *Corresponding author
}

(Received February 26, 2016; Accepted March 9, 2016)

\begin{abstract}
In this study, it is considered that the shape of membership function and the values of parameters are arbitrary, hence reliability parameters will be arbitrary, and some data are even absurd from the point of view of real life practice. An applied fuzzy reliability theory is applied to practical engineering problem. Method of fuzzy reliability evaluation are developed.
\end{abstract}

Keywords-fuzzy reliability, membership function, permitted wear fuzzy value.

\section{Introduction}

The random variable was adopted to describing the objective randomness of such variable, in the conventional reliability analysis. As one of the objective facts, fuzziness exists in every problem related to real life. Fuzzy reliability theory is a new branch, which combines reliability with fuzzy mathematics. It is more reasonable that some variables and constraints are considered to the fuzziness from the point of view of engineering practice. In the analysis of fuzzy reliability, for obtaining the concrete data of reliability, the shape and values of parameters of membership function are adopted as a certainty function, therefore, the self-contradictory exists. In the reference (Dong et al., 2004), the following point of views are pointed out: the books on Fuzzy Set Theory often describe membership function on a basis of Common Set Theory and its characteristic function, It is found that the method existed theoretical shortcomings of processing concept and definition absolutely.

Most of the recent references of fuzzy reliability are focused on enlarging their engineering applied range. In reference Biondini et al. (2004), a computational model for solving the engineering problems with random general stress-fuzzy general strength was presented. In reference (Handbook of Boeing, 1975), a fuzzy reliability evaluation of captive power plant maintenance scheduling incorporating uncertain forced outage rate and load representation was presented. In reference (Handbook of Russian airliner, 1974), a fuzzy reliability analysis of concrete structures under consideration of bands of values bounded between suitable minimum and maximum extremes was presented. In reference Jiang et al. (2004), a two-level fuzzy comprehensive decision process applied to solving the fuzzy resonance vibration failure zone and fuzzy strength failure zone was presented.

A small part of recent fuzzy theory book and fuzzy reliability papers were further explored for improving the determination of the membership function. In (Dong et al., 2004) based on the concepts of relative membership degree and membership function, the Engineering Fuzzy Set 
International Journal of Mathematical, Engineering and Management Sciences

Vol. 1, No. 1, 34-40, 2016

https://dx.doi.org/10.33889/IJMEMS.2016.1.1-004

Theory was explored. In (Jiang and Chen, 2005), approaches for establishing membership function safe event was discussed, and the approach of how to obtain the membership of the fuzzy safe event from the known membership function of fuzzy strength was also discussed, and an example was used to prove the feasibility of this model. In (Jiang and Chen, 2003), the type and method of selecting membership function of random parameter was given, but this method is only appropriate for a special problem. In (Chen, 1998), a method of objectively determining a kind of performance function membership function was presented, but they were based on some assumption and their feasibilities were illustrated only by an example.

\section{Available Data of Practicable Permitted Wear Fuzzy Set}

In this paper, the major data of permitted wear fuzzy set has been obtained on the basis of Chen (1995) and Wenting (2005). Chen (1995) provides the permitted minimum size of outer diameter of the critical worn shaft (bolt, dowel etc.), and permitted maximum inner diameter of the hole (lug, sleeve etc.) and the radial clearance. The maximum and minimum initial size of the shaft and the hole can be gained by the known margin tolerance. In this paper, we use a simple practical method to analyze the data. There are following three steps in this method. Firstly, we estimate the nominal diameter $D_{0}$ of the joint according to the initial data. Secondly, considering that the mean clearance is $\Delta_{0}$ while the margin tolerance is given, we let permitted wear value $W^{*}$ be permitted radial clearance $\Delta^{*}$ minus the initial clearance $\Delta_{0}$. Finally, we used the relative parameter $W^{*} / D_{0}$ as the synthetic fuzzy value. As such fuzzy values are different of the joints of different parts, they comprise the fuzzy set. Wenting (2005) given the following principle for all the joints: the applied clearance of hinge joint should not be more than the maximum clearance between the shaft and the hole with lower one degree accuracy in the same size joint. Because the tolerance system of Russia does correspond with the original tolerance system of our country, we adopt the original tolerance system and compute respectively with $2,3,5$ degree accuracy of sliding seat and $6,8,10,24,30$, $36,60,80$ millimeter as typical nominal diameter size according to the above principle. So we get another group of relative parameter $W^{*} / D_{0}$. The number of the two group data is 79 . All of them are given in the Table 1.

According to Table 1, most of the relative permitted wear value $W^{*} / D_{0}$ is not more than $1 \%$. For example, one datum which is less than $1 \%$ takes the $62^{\text {nd }}$ one $(62 / 79=78 \%)$; another datum which is not more than $1 \%$ takes the $67^{\text {th }}$ one $(67 / 79=85 \%)$; the datum which correspond to the median order (40) is $W^{*} / D_{0}=0.70 \%$.

Let $X=W^{*} / D_{0} . X$ is lies in the interval 0 to $2.1 \%$, which is equally divided into 10 parts. The number of the dots is placed in Table 2 .

From Table 1 and Table 2, following conclusion can be drawn. If the interval $0 \sim 2.1 \%$ is divided into three intervals as high accuracy, middle accuracy and low accuracy, therefore the interval with high accuracy lies in $0.039 \%-0.50 \%$, and the shape of the curve declines as a line; the interval with middle accuracy lies in $0.50 \%+-1.30 \%$, and the shape is normal curve without the tails; the interval with low accuracy lies in $1.30 \%+-2.10 \%$, and the shape is a parabola.

According to the curve coming from the abovementioned tables, the following explanations can be pointed out:

(a) In the interval with high accuracy, the curve of the permitted wear fuzzy value is a line that is higher firstly, then lower. The phenomenon is caused by the data coming from the fit quality with high reliability requirement. That is to say, in this interval, high data of 
International Journal of Mathematical, Engineering and Management Sciences

Vol. 1, No. 1, 34-40, 2016

https://dx.doi.org/10.33889/IJMEMS.2016.1.1-004

the permitted wear value are required more than low data.

(b) In the interval with middle accuracy, the curve of the permitted wear fuzzy value is normal. And the distribution of the data of the permitted wear value is relatively concentrative. The values that lie at the middle are more than that at the both sides from the middle dot.

(c) In the interval with low accuracy, the total number of the data of the permitted wear value is small. This value changes a little for the low need of the permitted wear value. The curve may be taken as a parabola and so on.

\begin{tabular}{|c|c|c|c|c|c|c|c|c|c|}
\hline Order & 1 & 2 & 3 & 4 & 5 & 6 & 7 & 8 & 9 \\
\hline $\mathrm{X}$ & 0.039 & 0.048 & 0.054 & 0.064 & 0.073 & 0.085 & 0.087 & 0.088 & 0.10 \\
\hline 10 & 11 & 12 & 13 & 14 & 15 & 16 & 17 & 18 & 19 \\
\hline 0.11 & 0.12 & 0.12 & 0.12 & 0.14 & 0.15 & 0.17 & 0.19 & 0.21 & 0.21 \\
\hline 20 & 21 & 22 & 23 & 24 & 25 & 26 & 27 & 28 & 29 \\
\hline 0.21 & 0.24 & 0.26 & 0.30 & 0.31 & 0.33 & 0.34 & 0.34 & 0.35 & 0.37 \\
\hline 30 & 31 & 32 & 33 & 34 & 35 & 36 & 37 & 38 & 39 \\
\hline 0.38 & 0.40 & 0.42 & 0.47 & 0.52 & 0.58 & 0.64 & 0.65 & 0.66 & 0.67 \\
\hline 40 & 41 & 42 & 43 & 44 & 45 & 46 & 47 & 48 & 49 \\
\hline 0.70 & 0.70 & 0.72 & 0.79 & 0.80 & 0.82 & 0.83 & 0.83 & 0.83 & 0.83 \\
\hline 50 & 51 & 52 & 53 & 54 & 55 & 56 & 57 & 58 & 59 \\
\hline 0.86 & 0.87 & 0.87 & 0.87 & 0.87 & 0.87 & 0.87 & 0.87 & 0.87 & 0.87 \\
\hline 60 & 61 & 62 & 63 & 64 & 65 & 66 & 67 & 68 & 69 \\
\hline 0.87 & 0.87 & 0.87 & 1.0 & 1.0 & 1.0 & 1.0 & 1.0 & 1.10 & 1.12 \\
\hline 70 & 71 & 72 & 73 & 74 & 75 & 76 & 77 & 78 & 79 \\
\hline 1.19 & 1.19 & 1.40 & 1.42 & 1.59 & 1.78 & 1.87 & 2.00 & 2.10 & 2.10 \\
\hline
\end{tabular}

Table 1. The statistics of fuzzy value $W^{*} / D_{0}$ (let $X=W^{*} / D_{0}$, unit: \%)

\begin{tabular}{|c|c|c|c|c|c|}
\hline Interval $/ \%$ & $0-0.21$ & $0.21_{+}-0.42$ & $0.42_{+}-0.63$ & $0.63_{+}-0.84$ & $0.84_{+}-1.05$ \\
\hline Number of data & 20 & 12 & 3 & 14 & 18 \\
\hline Interval $/ \%$ & $1.05_{+}-1.26$ & $1.26_{+}-1.47$ & $1.47_{+}-1.68$ & $1.68_{+}-1.89$ & $1.89_{+}-2.10$ \\
\hline Number of data & 4 & 2 & 1 & 2 & 3 \\
\hline
\end{tabular}

Table 2. Number of data in the sub-interval of $W^{*} / D_{0}$

\section{Confirmation of Membership Function}

The confirmation of the membership functions of the permitted wear value $W^{*} / D_{0}$ according to the three accuracy regulations are deduced. (In brief, the notion of $\chi$ stands for $W^{*} / D_{0}$, and $\mu(\chi)$ stands for the membership function).

\subsection{Interval with High Accuracy $(\chi=0.039 \%-0.50 \%)$}

Because the curve of the density function declines as a line, the density function can be got $f(\chi)=-$ $4121 x+23.6$. Integrating it, one gets $f f(x) \mathrm{d} x=-2061 x^{2}+23.6 x+c$; at $x=0.5 \%$, the integral of $f(x)$ is zero, so $c=-6.65 \times 10^{-3}$. In order that the integral value is one at $x=0.039 \%$, the integral function need to be magnified 17.35 times. Then, the integral function becomes the membership function $\mu(x)=35766 x^{2}-409.46 x+1.154$ (limited in the interval $0.039 \%-0.5 \%$ ). Therefore, the integrals of membership functions in the high accurate interval are:

$$
\begin{aligned}
& \mu(x)=1 \quad x \leq 0.039 \% \\
& \mu(x)=35766 x^{2}-409.46 x+1.154 \quad 0.039 \%<x \leq 0.5 \% \\
& \mu(x)=0 \quad x>0.5 \%
\end{aligned}
$$


International Journal of Mathematical, Engineering and Management Sciences

Vol. 1, No. 1, 34-40, 2016

https://dx.doi.org/10.33889/IJMEMS.2016.1.1-004

\subsection{Interval with middle accuracy $(\chi=0.50 \%-1.30 \%)$}

As the above, the membership functions in the middle accurate interval are:

$\mu(x)=1 \quad x \leq 0.5 \%$

$\mu(x)=1-1.017 \Phi[(x-0.0086) / 0.00163] \quad 0.5 \%<x \leq 1.3 \%$

$\mu(x)=0 \quad x>1.3 \%$

(Note: $\mu(x)$ in the interval $0.5 \%-1.30 \%$ accords with the normal distribution $N\left(0.0086,0.00163^{2}\right)$ verified by $\chi^{2}$ distribution)

\subsection{Interval with low accuracy $(x=1.3 \%-2.1 \%)$}

As the above, the membership functions in the middle accurate interval are:

$\begin{array}{lrl}\mu(x)=1.00 & x \leq 1.3 \% & \\ \mu(x)=2.633-127.6 \cdot x+152.4 x^{2}-47.8 x^{3} & 1.3 \%<x \leq 2.1 \% \\ \mu(x)=0 \quad x>2.1 \% & \end{array}$

(Note: $\mu(x)$ in the interval $1.3 \%-2.1 \%$ will accord with the stated express if the frequency is multiplied by 10.)

\section{Reliability Computation Formula \\ 4.1 Fuzzy Reliability Modeling}

According to conventional reliability, we set up safety boundary equation (or limit state equation): $W^{*}-W=0$

In that, $W^{*}$ is a permitted wear fuzzy value with membership function obtained by the equation (1), (2) or (3) according to various accurate requirement. $W$ is a practical wear random variable, and its distribution is generally considered as normal. Comparing with the conventional reliability computation, the fuzzy reliability computation needs the reliability multiplied by the membership value for every permitted wear value, and its proportion is related to the density distribution. So the fuzzy reliability can be gotten by numerical integral.

\subsection{Computation Formulas of Wear Fuzzy Reliability}

For the conventional reliability computation, the formula can be deduced by the safety boundary equation. Namely, if the signal $M$ stands for the flowing function of random variables: $M=W^{*}-W$. Here signal definition is the same as the above. Conventional reliability $R$ can be expressed:

$R=P(M>0)=\int_{0}^{\infty} M \mathrm{~d} M$

In that, $P$ stands for probability.

For the fuzzy reliability computation, the following steps will be used. Firstly, $X\left(X=W^{*} / D_{0}\right)$ is divided into many small blocks. For the middle point of the small block, $x_{i}$ stands for its horizontal coordinate and $f_{x_{i}}$ stands for its vertical coordinate. So the area of this small block is

$p_{i}=f_{x_{i}} \cdot \Delta x_{i} / 1$

Here, $\Delta x_{i}$ is the width of its block. For every small block, its fuzzy reliability is:

$\Delta R_{i}=C_{\mu} \cdot \mu\left(x_{i}\right) \cdot p_{i} \cdot R_{w_{i}^{*}}$

In that, $C_{\mu}$ is the mean magnifying coefficient, which is subject to $\bar{\mu}\left(x_{i}\right)=1$. In the process of fuzzy 
International Journal of Mathematical, Engineering and Management Sciences

Vol. 1, No. 1, 34-40, 2016

https://dx.doi.org/10.33889/IJMEMS.2016.1.1-004

reliability computation (Just as that of conventional reliability computation, the permitted wear value is considered as a certain quantity. Namely the safety degree at this value is $100 \%$ ), the mean of the permitted wear value is considered to be 1 , which implies that the mean safety degree is $100 \%$. So:

$$
C_{\mu}=\frac{1}{\sum_{i=1}^{n}\left(\mu_{i} / n\right)}
$$

Here, $n$ is the number of blocks. And

$R_{W_{I}^{*}}=\int_{0}^{\infty} M \mathrm{~d} M$

$M=W_{i}^{*}-W$

As $W$ is usually subject to the normal distribution and $W_{i}^{*}$ is a constant,

$R_{w_{i} *}=\Phi(\beta)=\Phi\left(\frac{W_{i}^{*}-\bar{W}}{\sigma_{\bar{W}}}\right)$

Here, $\Phi$ is cumulate standard normal distribution function. So the total fuzzy reliability is:

$\underset{\sim}{R}=\sum_{i=1}^{n} \Delta R_{i}=\sum_{i=1}^{n} C_{\mu} \cdot \mu\left(x_{i}\right) \cdot p_{i} \cdot R_{W_{I}^{*}}$

\section{Simulation}

In three situations of high accuracy, middle accuracy and low accuracy, let the mean of wear value $\bar{W}$ be $0.0009 D_{0}, 0.007 D_{0}$ and $0.012 D_{0}$ respectively, and the variance coefficient of the wear value be $V_{W}=0.065(\sigma=0.065 \bar{W})$. The aim is to calculate the reliability of fuzzy wear in these three situations.

By the model and the formulas built in the paper, the steps that we calculate the reliability of fuzzy wear in the middle accurate interval has shown in the following table:

\begin{tabular}{|c|c|c|c|c|c|c|c|c|}
\hline$X=W_{i}^{*} / D_{0}$ & 0.0055 & 0.0065 & 0.0075 & 0.0085 & 0.0095 & 0.0105 & 0.0115 & 0.0125 \\
\hline$R_{W^{*}}$ & 0.0005 & 0.1357 & 0.8643 & 0.9995 & 1 & 1 & 1 & 1 \\
\hline$P_{i}$ & 0.0408 & 0.1085 & 0.1982 & 0.2484 & 0.2137 & 0.1262 & 0.0511 & 0.0142 \\
\hline$\mu\left(x_{i}\right)$ & 0.9707 & 0.8980 & 0.7443 & 0.5213 & 0.2791 & 01081 & 0.0211 & 0 \\
\hline$C_{\mu}$ & \multicolumn{7}{|c|}{2.8228} \\
\hline $\begin{array}{c}\Delta R_{i} \\
\sim\end{array}$ & 0.0001 & 0.0373 & 0.3599 & 0.3653 & 0.1684 & 0.0385 & 0.0156 & 0 \\
\hline \begin{tabular}{c}
$R_{\sim}$ \\
\hline
\end{tabular} & \multicolumn{10}{|c|}{0.9851} \\
\hline
\end{tabular}

Table 3. Fuzzy reliability computation in interval with middle accuracy

The computation result in Table 3 implies that the reliability of fuzzy wear is 0.9851 in the middle accurate instance. By the same way, the reliability of fuzzy wear in high and low accurate instances is 0.9943 and 0.9771 respectively.

If we don't consider the fuzziness, one gets:

$R_{c}=\sum_{i=1}^{n} P_{i} \cdot R_{W_{i}^{*}} \cdot$ 
International Journal of Mathematical, Engineering and Management Sciences

Vol. 1, No. 1, 34-40, 2016

https://dx.doi.org/10.33889/IJMEMS.2016.1.1-004

The conventional reliability in those three instances: high accurate, middle accurate, low accurate is $0.8592,0.8395$ and 0.99997 respectively.

Compare the fuzzy reliability of wear $(R)$ with the conventional reliability $\left(R_{c}\right)$ of wear, let the relative difference $\varepsilon$ :

$\varepsilon=\left(R-R_{c}\right) / R$.

So, the value of it is $13.6 \%, 14.7 \%$ and $-2.3 \%$ in the relative instance, respectively.

\section{Conclusion}

From the result of the example, the fuzzy reliability gotten by the method that the membership function of the permitted wear value is decided by the practical permitted wear value, which is proposed in this paper based on the practical engineering experiment, is small different to the conventional reliability without considering fuzziness. The high, middle and low accuracy is respectively corresponding to $13.6 \%, 14.7 \%$ and $-2.3 \%$. So we know that the accuracy of the fuzzy reliability is higher than that of conventional reliability sometimes but sometimes not.

The deference between the fuzzy reliability and the conventional reliability comes from the deference of their membership functions used in the process of computation, which results in the deference of the item $C_{\mu} \mu_{i}(x)$. So the important problem in calculating fuzzy reliability is how to determine the membership function. If the fuzzy statistics is arbitrarily decided, then the computation has bigger deference. Of cause, the conventional reliability, which ignores the fuzziness of permitted wear value, is not advisable.

In the references Mohanta et al. (2004); Chen (1995); Jiang and Chen (2003); Jiang and Chen (2005), the further discussion of correct determination of membership function was discussed. Some theoretical exploring was presented by Mohanta et al. (2004), and in reference Chen (1995); Jiang and Chen (2003); Jiang and Chen(2005) the methods of determining the membership function for some special problems were investigated, but they were based on some assumption and their feasibilities were illustrated only each by one example. And the method presented by this paper is more reasonable by the engineering practical data foundation and a set of correct derivation without any assumption.

\section{References}

Biondini, F., Bontempi, F., \& Malerba, P. G. (2004). Fuzzy reliability analysis of concrete structures. Computers \& Structures, 82(13), 1033-1052.

Chen, S. Y. (1995). On absolutely membership degree and its determination. Discovery of Nature, 14(52), 19-22.

Chen, S. Y. (1998). Engineering fuzzy set theory and application. National Defence Industry Press, Beijing, 80-93.

Dong, Y. G., Chen, X. Z., Cho, H., \& Kwon, J. (2004). Fuzzy reliability digital simulation of mechanical system based on fuzzy theory [J]. Acta Simulata Systematica Sinica, 9, 028.

Handbook of Boeing 707-320B/C airliner maintaining. Beijing: Civil Aviation Beijing Administration System, 1975. 73-79.

Handbook of Russian airliner An-24 overhaul. Beijing: China Aviation, 1974. 103-107. 
International Journal of Mathematical, Engineering and Management Sciences

Vol. 1, No. 1, 34-40, 2016

https://dx.doi.org/10.33889/IJMEMS.2016.1.1-004

Jiang, L. P., Xu K.J. \& Sui, Y. S. (2004). Failure zone confirming of structure components based on fuzzy comprehensive decision process. Journal of Mechanical Strength, 26(1), 109-112.

Jiang, Q., \& Chen, C. H. (2003). A numerical algorithm of fuzzy reliability. Reliability Engineering \& System Safety, 80(3), 299-307.

Jiang, T. \& Chen, J. J. (2005). Assignment of membership function in profust reliability context. Fuzzy Systems and Mathematics, 19(3), 141-150.

Mohanta, D. K., Sadhu, P. K., \& Chakrabarti, R. (2004). Fuzzy reliability evaluation of captive power plant maintenance scheduling incorporating uncertain forced outage rate and load representation. Electric Power Systems Research, 72(1), 73-84.

Wenting, W. L. L. (2005). Model for structural fuzzy reliability analysis based on fuzzy random parameter [J]. Journal of Beijing University of Aeronautics and Astronautics, 31(4): 412-415. 